lying around the house; assisting a client with using a sharp; and disposing of sharps. Poisson regression modelling was used to identify important predictors of handling or encountering used sharps. By linking these results to national data on HC visits and clients, we estimated the frequency with which these hazardous conditions occur to $\mathrm{HC}$ aides nationwide.

Results Although not authorised to do so, $7 \%$ of aides assisted clients to use a sharp. Aides were much more likely to encounter sharps if they were employed directly by clients/families than if employed through an agency. Other important determinants of sharps exposure included client medical conditions like diabetes, and aide characteristics including professional certification.

Conclusions The results are being investigated further through focus groups of $\mathrm{HC}$ aides and used to develop preventive interventions.

\section{THE NIEHS GULF STUDY: QUESTIONNAIRE RESULTS AND USE OF JOB EXPOSURE MATRICES TO LINK INHALATION AND DERMAL EXPOSURE ESTIMATES TO STUDY SUBJECTS}

\begin{abstract}
${ }^{1}$ Patricia Stewart, ${ }^{2}$ Mark Stenzel, ${ }^{3}$ Sudipto Banerjee, ${ }^{4}$ Aaron Blair, ${ }^{5}$ John Cherrie, 6,7 Lawrence Engel, ${ }^{3}$ Caroline Groth, ${ }^{8}$ Tran Huynh, ${ }^{7}$ Richard Kwok, ${ }^{9}$ Wendy McDowell, ${ }^{5}$ Melanie Ng Gorman, ${ }^{8}$ Gurumurthy Ramachandran, ${ }^{6,7}$ Dale Sandler, ${ }^{5}$ Anne Sleuwenhoek. ${ }^{1}$ Stewart Exposure Assessments, LLC, Arlington, VA, USA; ${ }^{2}$ Exposure Assessment Applications, LLC, Arlington, VA, USA; ${ }^{3}$ Division of Biostatistics, University of Minnesota, Minneapolis, MN, USA; ${ }^{4}$ National Cancer Institute, Gaithersburg, USA; ${ }^{5}$ Institute of Occupational Medicine, Edinburgh, UK; ${ }^{6}$ Department of Epidemiology, University of North Carolina at Chapel Hill, Chapel Hill, NC, USA; ' Epidemiology Branch, National Institute of Environmental Health Sciences, Research Triangle Park, NC, USA; ${ }^{8}$ Department of Environmental Health Sciences, University of Minnesota, Minneapolis, MN, USA; ${ }^{9}$ MCDowell Safety and Health SVCS, LLC, Sanger, TX, USA
\end{abstract}

\subsection{6/oemed-2014-102362.114}

Objectives To describe the activities of the GuLF STUDY participants responding to the Deepwater Horizon oil release in the Gulf of Mexico in 2010 and the process of developing job exposure matrices (JEMs) of exposure group/location/time period combinations to link inhalation and dermal exposures to the participants.

Method Information on activities performed by the participants in the NIEHS epidemiologic study were collected via questionnaire with details on almost 100 clean-up activities (e.g., skimming); dates; amount of time spent performing these activities; and the geographic location where these activities were performed. The questionnaire also collected information on frequency of exposure to various oil components onto various parts of the body and the use of protective equipment. JEMs of inhalation and dermal exposure estimates have been developed for total hydrocarbons for unique exposure group/vessel/time period combinations.

Results Preliminary data indicate over 6000 study subjects reported patrolling the beaches, reported removing tar balls, and reported removing oil or oily sand. Over 5000 collected oily plants, a similar number bagged oiled material and over 4000 decontaminated vessels or equipment of oil. Approximately onethird of the study subjects worked on the water; about 5\% worked near the wellhead.

Conclusions Study subjects performed a variety of activities at multiple locations that resulted in different levels of inhalation and dermal estimates. These estimates will be used in the evaluation of exposure-response relationships in the epidemiologic study.

\section{BURDEN OF CANCER ATTRIBUTABLE TO OCCUPATIONAL DIESEL ENGINE EXHAUST EXPOSURE IN CANADA}

${ }^{1}$ Joanne Kim, ${ }^{2}$ Cheryl E Peters, ${ }^{2}$ Chris McLeod, ${ }^{3}$ Sally Hutchings, ${ }^{3}$ Lesley Rushton, ${ }^{1}$ Manisha Pahwa, ${ }^{1,4}$ Paul A Demers. 'Occupational Cancer Research Centre, Toronto, ON, Canada; 'University of British Columbia, Vancouver, BC, Canada; ${ }^{3}$ mperial College London, London, UK; ${ }^{4}$ University of Toronto, Toronto, ON, Canada

\subsection{6/oemed-2014-102362.115}

Objectives To estimate the number of new lung cancers cases in Canada attributable to occupational diesel engine exhaust (DEE), which IARC classified as a definite human carcinogen in 2012. This is part of a larger effort to estimate the current burden of occupational cancers in Canada.

Method Relative risks were selected from two recent studies of miners and truckers with quantitative exposure-response. CAREX Canada estimates of exposure prevalence and level by detailed industry and occupation were supplemented by a literature search for DEE measurement data. For each exposure group, RRs were assigned based upon the estimated mean exposure. Employment trends of industries and occupations were based upon census data from multiple years. Annual Labour Force Survey data were used to attribute age- and tenure-distribution, as well as short-term turnover characteristics. Survival was adjusted to age at entry into the exposed cohort during the risk exposure period 1961-2001. The attributable fraction (AF) for DEE-related lung cancers will be calculated by province, sex, industry and occupation.

Results Approximately 1.4 million workers were exposed to DEE during the risk exposure period. The initial estimated AFs for DEE-related lung cancers are: $4.92 \%$ for males, $0.29 \%$ for females, and $2.70 \%$ overall.

Conclusions These burden estimates are somewhat higher than recent estimates from other groups (1.3-1.8\%). They account for the most recent evidence for the risk of lung cancer from occupational DEE exposure, as well as detailed historical exposure assessment and labour force trends. Sensitivity analyses are underway to determine the influential assumptions.

\section{DEVELOPMENT OF A PREDICTIVE MODEL FOR ESTIMATING GAMMA RADIATION EXPOSURES AMONG ONTARIO URANIUM MINERS}

Minh Do. Occupational Cancer Research Centre, Toronto, Ontario, Canada

\subsection{6/oemed-2014-102362.116}

Objectives The objective of this study is to develop and validate a predictive model for estimating gamma radiation exposure for miners working in uranium mines between 1981 and 1985 .

Method The dose prediction model was developed and validated using multiple linear regression. To aid in model development, $70 \%$ random sample of workers were used in the model development (i.e., Training Sample) while the remainder 30\% (i. e., Test Sample) was used to determine model performance. A stepwise approach was used to select variables into the model. Criteria for retaining the variables in the model was based on a $\mathrm{p}$-value of $<=0.15$. Model fit was assessed using adjusted Rsquare. Co-linearity was determined by the magnitude of the variance inflation factor (VIF). Variables with VIF greater than 3.0 were removed from the model. In addition, SAS procedure ROBUSTREG was used to minimise the effects of outliers and 
high leverage in order to provide resistant (stable) results in the presence of outliers and high leverage.

Results Based on 8949 employments records, dosimetric measurements of gamma radiation were significantly correlated with radon exposure $(r=0.499)$, duration of employment $(r=0.429)$, year of exposure $(r=0.239)$, and ore production $(r=0.230)$. Age was inversely related to gamma dose. Regression analysis showed that individual dosimetric readings can be modestly predicted by individual work history and geological characteristics of Ontario uranium mines $(\mathrm{p}<0.001, \mathrm{R} 2=0.374)$. Additional sources of variation are likely related to individual variability that could not be accounted for in this ecological assessment.

Conclusions Reconstructed gamma dose provides modest agreement with individual dosimetric readings.

\section{AN ASSESSMENT OF THE IMPACT OF MISCLASSIFICATION ERROR ON AN ESTIMATE OF EFFECT FOR OCCUPATIONAL EXPOSURE TO TCDD- CONTAMINATED CHEMICALS AND ISCHAEMIC HEART DISEASE}

Laura Scott. University of Minnesota School of Public Health, Minneapolis, MN, USA

\subsection{6/oemed-2014-102362.117}

Objectives To quantify the effect of exposure and disease misclassification on the odds ratio (OR) for ischaemic heart disease (IHD) mortality in a retrospective cohort study of trichlorophenol workers exposed to 2,3,7,8-tetrachlorodibenzo-p-dioxin (TCDD).

Method Exposure and disease classification parameters were defined using specificity and sensitivity values either reported in the peer-reviewed literature or calculated from available published data. The distribution shapes for each parameter were constructed based on expert judgment and were varied to address the lack of published information on the parameter distributions. Probabilistic uncertainty analysis, which uses Monte Carlo simulation techniques, was then used to sample each parameter distribution, calculating ORs corrected for outcome and exposure misclassification.

Results Correction for exposure and disease misclassification produced lognormal probability distributions of ORs for IHD with a wider range of possible values than calculated in the traditional epidemiological analysis. The median $\mathrm{OR}_{\text {corrected }}$ was larger than the $\mathrm{OR}_{\text {observed }}$ of 3.05 for all seven scenarios evaluated and ranged from 5.34 to 11.86 . Between $70 \%$ and $99 \%$ of the simulation trials yielded corrected ORs greater than the $\mathrm{OR}_{\text {observed }}$.

Conclusions The application of uncertainty analysis to a mortality study of workers occupationally exposed to TCDD-contaminated chemicals provides valuable insight into the magnitude and direction of misclassification error and the impact on an estimate of effect. Further refinement of the parameter distributions and adjustment of the $\mathrm{OR}_{\text {observed }}$ for other study limitations will be necessary to determine whether a true causal relationship between exposure and disease exists or if the effect observed in these workers is an artefact of systematic error.

\section{USING META-DATA FROM OCCUPATIONAL STUDIES TO INFORM HAZARD IDENTIFICATION AND CANCER CONTROL: THE IARC MONOGRAPHS AND BEYOND}

Neela Guha, Dana Loomis, Kurt Straif. IARC, LYON, France

10.1136/oemed-2014-102362.118
Objectives To present extensions to the International Agency for Research on Cancer (IARC) Monographs process, providing examples of application of meta-data to identifying carcinogenic hazard identifications and research gaps, and the potential use for guiding cancer control efforts. Qualitative and quantitative approaches will be contrasted.

Method The IARC evaluation process typically employs summary level meta-data, in the form of systematic reviews, and pooled- and meta-analyses.

Results IARC has heavily relied on published occupational epidemiological studies to identify specific carcinogens in the workplace and to form a scientific basis for the protection of workers worldwide. The evaluations of carcinogenic risk are made by international working groups of independent scientists and are qualitative in nature. Meta-analyses prepared for IARC working groups can complement the qualitative process and have been crucial in several instances; for example, in identifying sufficient evidence for lung and bladder cancer in painters and limited evidence for increased risk of bladder cancer among dry cleaners exposed to tetrachloroethylene and among professional drivers (bus, taxi, truck) with high exposure to outdoor air pollution. Since IARC does not provide recommendations for regulation or legislation, meta-relative risks can also be used to calculate attributable fractions to guide cancer control efforts, for agents in which a causal association is assumed and exposure prevalence has been well-characterised.

Conclusions The IARC Monographs Programme is an authoritative source for the identification of carcinogenic hazards in the environment. Applying meta-analyses to the IARC process can be a useful tool for informing hazard identification and providing guidance for cancer control efforts.

\section{ESTIMATING PERSONAL EXPOSURE TO TRAFFIC- RELATED AIR POLLUTANTS AMONG ON-ROAD WORKERS}

Kirsten Almberg, Jordan Hepler, Margaret Sietsema, Matthew Schmitz, Lorraine Conroy. University of Illinois at Chicago, Chicago, IL, USA

\subsection{6/oemed-2014-102362.119}

Objectives Many individuals in the U.S. work in close proximity to traffic-related air pollution. Often these jobs require physical activity which increases breathing rate and results in higher personal exposures to pollutants. The goal of this study was to systematically estimate and characterise personal exposure to traffic-related pollutants for those working on or near roadways. Method Concentrations of elemental carbon, carbon monoxide, and PM2.5 were measured along six traffic routes in the City of Chicago. These routes represent varying levels of exposure to traffic-related pollutants. Measurements were taken during peak and off-peak traffic hours during the summer and fall. All measurements were obtained from environmental monitoring equipment affixed to the back of a bicycle.

Results Air pollutant levels varied significantly across routes and time of day. Mean carbon monoxide levels ranged from 0.006$1.653 \mathrm{ppm}$ across the sampling events. The geometric mean elemental carbon level was $1.75 \mu \mathrm{g} / \mathrm{m}^{3}$ and ranged from 0.23 to $8.38 \mu \mathrm{g} / \mathrm{m}^{3}$. The geometric mean $\mathrm{PM}_{2.5}$ level was $39 \mu \mathrm{g} / \mathrm{m}^{3}$ and ranged from 16 to $270 \mu \mathrm{g} / \mathrm{m}^{3}$. Levels of elemental carbon and $\mathrm{PM}_{2.5}$ were significantly higher during peak traffic samples than off-peak traffic samples.

Conclusions This study uses novel methods to estimate exposure to those who are physically active along roadways including 